DOI: https://doi.org/10.11144/Javeriana.upsy17-1.tbvm

\title{
Tareas de búsqueda visual: modelos, bases neurológicas, utilidad y prospectiva*
}

\section{Visual Search Tasks: Models, Neurological Bases, Usefulness and} Prospective

Recepción: 02 Diciembre 2015 | Aprobación: 03 Junio 2017

\author{
Guillermo Andrés Rodríguez Martínez \\ Universidad de Bogotá Jorge Tadeo Lozano, Colombia \\ ORCID: http://orcid.org/0000-0003-4329-5745 \\ Henry Castillo Parra \\ Universidad de San Buenaventura de Medellín, \\ Colombia
}

a Autor de correspondencia. Correo electrónico: guillermo.rodriguez@utadeo.edu.co

Para citar este artículo: Rodríguez, G., \& Castillo, H. (2018). Tareas de búsqueda visual: modelos, bases neurológicas, utilidad y prospectiva. Universitas Psychologica, 17(1), 1-12. doi: https://doi.org/10.111 44/Javeriana.upsy17-1.tbvm

\section{RESUMEN}

Las tareas de búsqueda visual, fundamentadas en el reconocimiento de una forma, característica o estímulo visual en particular, han contribuido al desarrollo de la investigación en psicología. A partir de una revisión narrativa sobre las maneras de implementar dichas tareas, y haciendo un cotejo de su uso como elemento de instrumentalización de la investigación, se encuentra que diferentes modelos paradigmáticos convergen para el entendimiento de diversos procesos psicológicos. Se hace manifiesto que las tareas de búsqueda visual, no solo pueden implicar registros de actividad oculo-motora, sino que además operacionalizan sistemas de modulación de procesos atencionales y perceptuales, los cuales esencialmente están alineados con recompensas de tipo exógeno. Dichas recompensas, sumadas a procesos de adaptación al entorno, se integran para reivindicar la noción percepción para la acción.

\section{Palabras clave}

tareas de búsqueda visual; percepción; procesamiento visual; movimientos oculares; atención.

\section{ABSTRACT}

Visual search tasks, that are based on the recognition of either a characteristic or an specific visual stimulus, have contributed to the development of psychological research. By doing a narrative review of the ways to carry out these tasks, and considering their usages as factors that instrumentalise research, several paradigms emerge so as to understand multiple psychological processes. It has been manifested that visual search tasks can imply eye-movement recordings as well as operationalizing systems connected to the modulation of both attentional and perceptual processes, which are essentially aligned with exogenous rewards. These rewards, added to some processes related to the adaptation to the environment, are incorporated in such a way that the notion of perception to action is enforced.

Keywords

visual search tasks; perception; visual processing; eye movements; attention. 
Las tareas de búsqueda visual se basan en la localización específica de un determinado objeto o estímulo que se encuentra entre un grupo de distractores. De conformidad con los objetivos que en específico tenga uno $\mathrm{u}$ otro estudio, las tareas de búsqueda visual se inscriben dentro de diferentes modelos, donde factores como los tiempos de búsqueda del objetivo, la identificación de características en específico de los estímulos visuales, la configuración de mapas de características por dimensiones a codificar, los efectos de saliencia atencional y los patrones conductuales asociados a la actividad motora de los ojos, entre otros, pueden ser estimados. Resulta frecuente encontrar que los participantes que realizan este tipo de tareas son previamente instruidos y/o capacitados, de suerte tal que entienden lo que en específico deben hacer y el tipo de acción (reporte verbal, pulsación de una tecla, parpadeo, etc.) que da cuenta del reconocimiento o detección del objetivo. Desde esta perspectiva, aspectos como los tiempos de reacción, la taza de aciertos o desaciertos frente a la tarea planteada, o la capacidad de discriminación de características intrínsecas de los estímulos, se contemplan y, a su vez, abonan en la interpretación que el investigador hace de los resultados, previo establecimiento del paradigma y marcos referenciales.

El presente artículo, fundamentado en una revisión narrativa sobre el rastreo visual de imágenes, recoge diferentes modelos paradigmáticos que convergen en el entendimiento de la percepción visual como un detonante de las acciones humanas, donde las tareas de búsqueda visual se alinean tanto con medidas y registros de actividad oculomotora, como con sistemas de modulación de procesos atencionales y perceptuales que preponderantemente responden a recompensas de tipo exógeno.

\section{Método}

Para efectos de realizar la revisión narrativa sobre el uso de las tareas de búsqueda visual, sus usos e implicaciones, se hizo un rastreo de fuentes utilizando las bases de datos EBSCO, PubMed, Science Direct y Scopus. Los descriptores contemplados fueron "visual search tasks", "serial search tasks", "visual processing", "attentional processes", "searching for visual targets", "visual perception", "eye-tracking studies" and "visual research paradigms". Adicionalmente, se recurrió a una búsqueda manual que derivó en cinco libros con apartados igualmente relacionados con los descriptores. Fueron considerados los textos que refirieran factores metodológicos, técnicos y/o de paradigmas de investigación, con referencia a estudios sobre percepción visual y atención visual. Mediante procedimientos automáticos y manuales (combinadamente), se identificaron fuentes repetidas, las que, acto seguido, fueron debidamente removidas. Esta labor de búsqueda se realizó durante el segundo semestre de 2015, y permitió obtener más de 45 referencias, incluyendo algunos capítulos de libro, artículos científicos y volúmenes alusivos a las nociones sensación y percepción. Adicional a la inclusión de textos que hicieran referencia a modelos paradigmáticos, se consideraron artículos que contuvieran tareas experimentales asociadas a búsquedas de objetivos visuales, y que implicaran procesos perceptuales mediados por procesamientos bottom-up y top-down.

\section{Resultados}

Registro de movimientos oculares dentro de la investigación psicológica

Los movimientos oculares han sido objeto de estudio para efectos de comprender diferentes actividades de orden cognitivo referidas a la atención, la percepción y el aprendizaje, preponderantemente. De conformidad con los planteamientos hechos por Rayner (1998), la investigación centrada en los movimientos oculares como objeto de estudio ha encontrado tres períodos históricos fundamentales, entendidos como las tres eras de la investigación en este campo. De manera 
esencial, se dirimieron estos tres períodos desde criterios referidos a las aportaciones hechas en el campo de estudio, en relación con una evolución tanto conceptual como instrumental de la investigación sobre los movimientos de los ojos. Así, de 1879 a 1920, se consolidó lo que se conoce como la primera era, donde el abordaje de los movimientos oculares se centró en comprender su papel en la lectura de textos (hacia 1879). También, dentro de este primer período, emergieron categorías que hasta nuestros días son relevantes, como la supresión sacádica (referida a la percepción nula de información durante el movimiento del ojo), la latencia del movimiento sacádico (el tiempo que toma el inicio de un movimiento ocular) y el tamaño del área abarcada perceptualmente (es decir, la región de visión efectiva, conocida como size of the perceptual span).

La segunda era se constituye por el período abarcado entre 1920 y la mitad de los setenta. Tomando como referente lo escrito por Rayner (1998), no fueron muchos los avances dados desde la investigación en este período que, como era de suponerse, tuvo aportaciones clave, especialmente en lo referente a preocuparse por indagar sobre el efecto de los movimientos oculares en los procesos cognitivos. Llegado el año 1976, momento en el que da inicio la tercera era en investigación sobre movimientos oculares, empezaron a darse saltos importantes de conformidad con los avances tecnológicos en cuanto al desarrollo de instrumentos, donde ya no se hacía necesario introducir elementos lectores invasivos dentro del ojo humano, puesto que se aportó en la manera de hacer un recording más preciso y controlado de los movimientos oculares desde el uso de lectores externos.

Siendo los movimientos oculares esenciales dentro del entendimiento de los parámetros de búsqueda visual, los investigadores en esta área, con consciencia de que sin un acertado control de variables, ciertos resultados se tomarían como espurios, y lograron decantar técnicas y paradigmas, simplificando al máximo la estimulación visual en comparación con el campo visual natural ecológico de la cotidianidad, todo esto implicado en tareas de movimientos sacádicos controlados (Brouwer, Reuderink, Vincent, van Gerven, \& van Erp, 2013; Dandekar, Ding, Privitera, Carney, \& Klein, 2012; Kazai \& Yagi, 1999), o en tareas de búsqueda visual con estimulación artificial (Kamienkowski, Ison, Quiroga, \& Sigman, 2012). De igual manera, el abordaje científico de la percepción visual derivó en el entendimiento de decisiones y conductas, que son muchas veces, la consecuencia de configuraciones perceptuales que actúan como moduladores frente al modo de interactuar con la realidad. Así, la preocupación por la maximización de la eficacia de las acciones humanas, se ha convertido en un elemento constante en el estudio del ser humano y su naturaleza, al punto de que se ha reconocido que la transformación del mundo en representaciones perceptuales remite a una traducción en parámetros motrices que buscan la optimización de la eficacia ya referida, de manera tal que se alude a una percepción para la acción, noción que para algunos es relativamente novedosa, y que reviste un punto a seguir para proponer investigaciones que no necesariamente encuentren aplicaciones inmediatas (Roca, Rosselló, Maiche, García, \& Roberts, 2008).

Desde el año 2000 en adelante, un creciente número de estudios han mostrado lo importantes que resultan ser ciertos aspectos propios de los observadores, especialmente factores referidos a orientar búsquedas de estímulos visuales con base en manifestaciones de la memoria implícita a corto plazo. Estas evidencias han sido remarcadas mediante experimentos intersujeto en diversas tareas de búsqueda visual (Found \& Müller, 1996; Geyer, Müller, \& Krummenacher, 2006; Maljkovic \& Nakayama, 2000; Müller, Heller, \& Ziegler, 1995; Treisman, 1988; Weidner, Pollmann, Müller, \& von Cramon, 2002). Desde esa perspectiva, resulta común entender por qué se hace investigación sobre procesamientos perceptuales top-down, efecto de congruencia semántica, modulación atencional, configuración y aprendizaje perceptual, distribución de la focalización atencional, entre otros. También ha sido relevante en el contexto científico, 
propugnar por entender qué procedimientos neurales se activan cuando el perceptor está ante una situación que lo lleva a buscar un determinado estímulo dentro de un conjunto, lo que, de entrada, presupone la posibilidad de modular el proceso atencional.

Al hacer una revisión de las aportaciones dadas por Trommershäuser, Glimcher, y Gegenfurtner (2009), se advierte que los movimientos oculares guardan relación con el procesamiento neural de información precedente, que puede interferir con el rastreo visual de imágenes y de estímulos visuales en general, de tal suerte que la implicación de recompensas exógenas, por ejemplo, puede suscitar un efecto tanto en los procesos atencionales como en los modos en que se hacen configuraciones perceptuales. En efecto, existe evidencia que indica que los movimientos oculares realizados durante tareas específicas de búsqueda visual implican cálculos neuronales similares, en alguna medida, al tipo de cálculos que se dan cuando los movimientos oculares pueden ser planificados (o, más bien, predeterminados), y también cuando existe la presencia de un motivador (modulador de la atención), esto es, de una recompensa dada a un sujeto con el ánimo de premiar el éxito en una tarea referida a la búsqueda de un estímulo en particular. Este hecho da emergencia a la idea de que información suministrada con anterioridad a la exposición de un determinado estímulo, implica un condicionamiento en la labor tanto atencional como perceptual, hecho que puede remitir a nociones esenciales dentro de la teoría de la percepción, como el procesamiento perceptual de tipo top-down, la modulación de la percepción y el aprendizaje perceptual, entre otras. Así, diversos estudios han sugerido la idea de que tanto el procesamiento topdown como el bottom-up implican un efecto en la percepción. Por ejemplo, investigaciones realizadas para estudiar el fenómeno de la reversibilidad perceptual de figuras ambiguas o biestables, dan cuenta de la importancia de los dos tipos de procesamiento (Hochberg \& Peterson, 1987; Kornmeier \& Bach, 2012; Leopold \& Logothetis, 1999; Long \& Toppino,
2004; Long, Toppino, \& Kostenbauder, 1983; Gale \& Findlay, 1983; Hsiao, Chen, Spence, \& Yeh, 2012). Evidencias de la repercusión de los procesamientos top-down y bottom-up han sido reportadas también cuando en la revisión de la reversibilidad perceptual de este tipo de figuras (biestables), se presenta a los sujetos, por un tiempo, una imagen que no acepta reversibilidad perceptual, para inmediatamente después exponer la figura biestable, de modo tal que el aprendizaje obtenido con la revisión de la primera aporta en la percepción del percepto alternativo de la imagen ambigua, lo que supone un efecto de adaptación (Long \& Moran, 2007; Long \& Olszweski, 1999; Long, Stewart, \& Glancey, 2002; Long, Toppino, \& Mondin, 1992; von Grünau, Wiggin, \& Reed, 1984).

Desde la información aunada por Trommershäuser et al. (2009), el tema de los movimientos sacádicos oculares (MOS) fácilmente se puede entender como un modelo paradigmático idóneo para indagar sobre los mecanismos conductuales y neuronales implicados en los procesos perceptuales (desde decisiones tanto de orden voluntario como involuntario). El abordaje de este tipo de categorías ha permitido sugerir una revaloración paradigmática enmarcada en la consideración de modelos computacionales que permitan explicar lo observado en experimentos fundados en tareas que guardan relación con los MOS. En esa línea de ideas, las aportaciones otorgadas por David Marr, pionero en el estudio de la percepción visual desde un enfoque computacional, han sumado a la discusión de si efectivamente se puede concebir la idea de mecanismos de orden matemático, que empleen los sistemas perceptuales para el procesamiento de estímulos visuales. Recuérdese que Marr, desde su enfoque, había planteado que los estímulos, que las formas de energía exteriores son ricas en información, y que diversos principios físicos operan dentro del proceso perceptual. Yendo directamente al texto de Marr (1982, p. 27), se encuentra una alusión directa a la importancia del estudio de la percepción desde el enfoque computacional, cuando, palabras más palabras menos, se expresa que la naturaleza 
de los cálculos que subyacen a la percepción depende más de los problemas computacionales que tienen que ser resueltos, que sobre el hardware particular en el que se implementan sus soluciones. Sin que se pretendan desestimar otros enfoques, la investigación cuyo objeto de estudio es la percepción visual y los movimientos oculares (en correlato con toma de decisiones, procesos de memoria y aprendizaje), y que busca encontrar predictores desde la lectura de cálculos neuronales implicados en ciertos procesamientos controlados en laboratorio, ha encontrado un espacio importante, especialmente si se toman en consideración los avances que desde ella se han dado en la ciencia básica y, posteriormente, en la aplicada.

Volviendo al paradigma fundado en el abordaje de los movimientos sacádicos, se han encontrado, por ejemplo, estudios tanto en monos como en seres humanos, que dan cuenta de la presencia de ciertos cálculos de orden neural relacionados con la selección de objetivos predefinidos, selección que está asociada con la iniciación de los movimientos oculares. Desde evidencia empírica, investigaciones soportan la idea de modelos computacionales presentes en tareas que implican la búsqueda de determinados estímulos, donde los MOS cumplen un rol esencial. En efecto, es plausible presentar un modelo descriptor del cerebro del primate que dé cuenta de una programación de los movimientos oculares sacádicos, tomando como referente movimientos oculares esperados (con uso de predictores) con una actualización de ellos fundamentada en un refuerzo positivo, dado a posteriori, esto es una recompensa, una retroalimentación que, en últimas, constituye un efecto de redundancia (Trommershäuser et al., 2009), similar al evento manifestado en procesos de comunicación (también efecto de redundancia), por el cual, según Maarek (2009), un emisor cambia su mensaje original de conformidad con el feed-back que da la audiencia sobre ese mensaje.

Haciendo eco de las descripciones hechas a propósito de la ruta que emplea la información visual, desde la retina hasta los centros corticales especializados en el procesamiento visual, se da el correspondiente arreglo retinotópico, en donde se estima que existen enlaces o conexiones paralelas entre el núcleo geniculado lateral y el sistema oculomotor mediante conectores presentes en el colículo superior (Wurts \& Goldberg, 1989). Así, se hace inmediata referencia a la generación de los movimientos sacádicos express. Estos movimientos en particular tienen una latencia de entre 80 y 120 milisegundos. Si bien desde diferentes autores la clasificación de los movimientos oculares puede tener ligeras variaciones de orden terminológico, es importante relacionar que, en esencia, los movimientos sacádicos express se pueden categorizar en tres grandes grupos: 1 . Automáticos, referidos a la compensación de los movimientos de la cabeza (reflejos vestíbulooculares) y del entorno (reflejos optocinéticos) para estabilizar la imagen de la retina y posibilitar la fijación, de forma voluntaria, de la mirada en un determinado punto; 2. Voluntarios, cuya particularidad es tener la función tanto de desplazar la fijación de un punto a otro en el campo visual (movimientos de refijación, sacádicos y sacadas), como de perseguir objetos en movimiento con la mirada (movimientos de seguimiento y vergencia); y, 3. Micromovimientos, asociados a la fijación ocular: temblor, microsacadas, derivas y nistagmos (Pires, Vásquez, Carboni, \& Maiche, 2014).

\section{Tareas de búsqueda visual: paradigma clave para el estudio del reconocimiento y tamizado perceptual de estímulos visuales}

Desde el punto de vista procedimental, el paradigma más frecuente para estudiar neuralmente procesos de selección de estímulos visuales es el de tareas de búsqueda visual. Es común que dentro de este tipo de diseño, los participantes sean debidamente instruidos y tengan que oprimir algún botón o tecla tan pronto como detectan el objetivo, el cual normalmente está entre un conjunto de estímulos distractores. Para efectos de valorar la información recolectada por esta vía, se toma en 
consideración que tanto los tiempos de reacción como los porcentajes de aciertos frente a la tarea en específico adjudicada, varían dependiendo del tamaño o características del conjunto de estímulos visuales presentados, de la similitud o disparidad entre el objetivo y los distractores, y en coherencia con lo anterior, de la posibilidad que tenga el estímulo objetivo de ser discriminado de entre el conjunto presentado (Treisman, 1991). Derivado de estas ideas, White, Stritzke, y Gegenfurtner (2008) proponen que los movimientos de latencia sacádica son mucho más cortos cuando un estímulo objetivo es expuesto a ambientes ruidosos, sin uniformidad y con desorden, que cuando se presenta en lugares con fondos uniformes. Esta idea está relacionada con un enfoque que explica que existen unas fases en el proceso de configuración perceptual: el enfoque de integración de las características. Anne Treisman (1986) planteó este modelo, en el que, en una primera instancia, emergen registros automáticos de las características, en donde se configura un procesamiento en paralelo, donde múltiples objetivos son procesados de manera simultánea (procesamiento paralelo por campo visual). Así, de manera sucesiva emerge una atención enfocada, a la cual le compete hacer distinciones e identificaciones de los estímulos de una manera seriada (lo que supone un procesamiento en serie de estímulos independizados, esto es, disgregados del conjunto). La Figura 1 muestra el modelo que explica la teoría de integración de las características: en una etapa pre-atentiva se hace una identificación de las formas primitivas, esto es, unidades básicas y simples: curvatura, orientación, líneas, color, movimiento, áreas cerradas, contraste y brillantez; en una etapa de atención concentrada, las formas primitivas se combinan y generan una configuración que permite percibir el objeto; por último, en un acto de comparación con lo registrado en la memoria, se sobreviene el acto de la identificación del objeto.
Figura 1

Modelo explicativo de la teoría de integración de las características

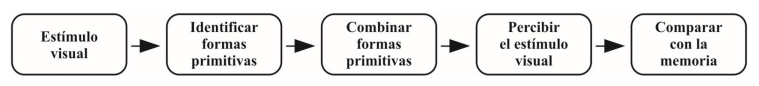

Fuente: Adaptación a partir de los planteamientos de Treisman (1986).

El postulado de la integración de las características claramente se sustenta en la ley gestáltica de la figura-fondo, puesto que se hace presente el establecimiento de relaciones de preponderancia entre los estímulos captados sensorialmente, advirtiendo momentos perceptuales en los que de la generalidad se pasa a la especificidad y viceversa, y donde se tiene en claro cuál es el fondo y cuáles las figuras, cuál el complejo integrado y cuáles las partes constitutivas. La filtración, concepto propio de las teorías explicativas de los fenómenos atencionales, induce las ideas atrás referidas, dado que subyace en el proceso, un tamizado entre un entramado de estímulos, dejando un único estímulo en el primer plano de la atención, esto es, permitiendo que un único canal de información sobresalga con respecto a la exposición general de estímulos (Coren, Ward, \& Enns, 2001). Esta filtración implica el concepto de orientación, que hace referencia a la acción de dirigir los receptores sensoriales hacia un conjunto de estímulos y desligarlos de otros. Debe considerarse que existe una orientación abierta, referida a la focalización hacia estímulos específicos por mediación de un movimiento corporal de los receptores, y una encubierta, que supone el cambio de la atención de un estímulo $\mathrm{A}$ a uno $\mathrm{B}$, sin que haya ningún movimiento corporal acompañante que indique el redireccionamiento focal (Coren et al., 2001). En suma, la organización que se hace de los estímulos visuales en función de su reflectancia en un campo visual, se genera por diferentes procesos, en donde cada uno opera a una escala diferente (Marr, 1982).

De manera general, se advierte que las tareas de búsqueda visual implican una actividad psíquica que parte de lo atencional, y que concluye en un reconocimiento de una forma 
o imagen, en particular, donde pueden mediar, como ya se había mencionado, aspectos como recompensas explícitas dadas por lograr un reconocimiento de imagen específico, o como el fenómeno del aprendizaje perceptual (o perceptivo), que fundamentalmente se centra en la idea de producir una adaptación al entorno para saber responder ante los cambios que se dan en él. Esto permite referir que uno de los componentes esenciales en cualquier tarea de acción de búsqueda es el direccionamiento que tiene la atención hacia el objetivo visual que se ha definido, donde encontrar dicho objetivo con el menor desgaste de recursos posible (tiempo, energía, procesamiento neural y cognitivo, etc.) supone una mayor eficacia y una mejoría progresiva desde la interpelación del aprendizaje (Xu \& Yue, 2014).

Desde un punto de vista neurofisiológico, el aprendizaje referido al reconocimiento de estímulos específicos guarda relación con la actividad neural manifestada en la corteza visual primaria, una vez recibe información del núcleo geniculado lateral. En el interior de la corteza visual primaria, módulos individuales de células analizan la información procedente de regiones delimitadas de la escena visual referentes a movimiento, orientación, color, disparidad binocular y frecuencia espacial. Debe estimarse que la información referida a cada uno de dichos atributos, se relaciona con subregiones de la corteza extraestriada, la que a su vez rodea a la corteza visual primaria (Carlson, 2006). Luego de que se haga un análisis de los atributos específicos de la escena visual, las subregiones de la corteza extraestriada envían el resultado de su análisis al siguiente nivel de la corteza visual de asociación. Así es como prácticamente inicia el recorrido de la información a nivel de la corriente ventral, que, como se sabe, es la que está implicada en el reconocimiento de objetos y que se dirige, luego del paso por la corteza extraestriada, hacia la corteza temporal inferior. De forma tal que, como se ha demostrado, lesiones cerebrales en la corteza inferotemporal impactan negativamente en la capacidad de discriminar entre diferentes estímulos visuales. Esto debe también entenderse considerando que pueden existir diferencias en los patrones de búsqueda visual según el género de la persona (Shah, Prados, Gamble, De Lillo, \& Gibson, 2013).

Nótese, entonces, cómo las tareas de búsqueda visual encuentran un pilar en el flujo de información manifestado en la corriente ventral (lo que no exime el papel igualmente crucial que tiene la otra vía, la dorsal, puesto que esta remite a la posibilidad de entender dónde está localizado un determinado estímulo entre un conjunto de estímulos, para lo que, como es sabido, es esencial la actividad del córtex parietal posterior). De hecho, cuando se alude a tareas de búsqueda visual por procedimientos de memoria guiada, tal y como lo manifiestan Thomas \& Pare (2007) y Glimcher (2009), se producen correlatos neurales de la actividad presacádica en el área lateral intraparietal (LIP). Así, se ha configurado otro paradigma para abordar el fenómeno de la búsqueda de estímulos específicos: el paradigma de movimientos sacádicos por memoria guiada. En esencia, este paradigma alude a que, una vez se ha presentado un estímulo de manera seguida, se da un período de retardo de unos pocos segundos hasta el inicio del movimiento del ojo.

Otro modelo importante para estudiar el fenómeno atencional y perceptual fundamentado en el reconocimiento de formas es el modelo bayesiano de búsqueda visual óptima (Nejemnik $\&$ Geisler, 2008). Este se funda en la idea de un modelo estratégico óptimo cimentado en la maximización de la información obtenida a través de sucesivos movimientos oculares. El modelo inicia con la imputación de posibles hipótesis sobre la localización probable del estímulo objetivo. Acto seguido, se da una codificación de la imagen donde se presenta una actualización de las primeras hipótesis de localización con otras subsiguientemente generadas como consecuencia del rastreo general del campo visual. En modo práctico, el modelo bayesiano sugiere un procesamiento eficiente de la información visual mediante la selección de posiciones de fijación que permiten maximizar dicho procesamiento, donde algo fundamental es lo que dentro del modelo se plantea como la 
inclusión de una inhibición del retorno, esto es, una capacidad para recordar y procesar información a través de una sucesión de fijaciones. Así, la memoria de trabajo incursiona como actante esencial dentro de la tarea de búsqueda visual, derivando en que, por inhibición del retorno, se impida que se hagan fijaciones repetidas sobre objetivos previamente fijados. En ese orden de ideas, la inhibición de retorno emerge como un componente de atención estratégica que opera de manera preponderante durante las tareas de búsqueda visual. En esa misma línea de ideas, Trommershäuser et al. (2009) hacen referencia a la inhibición del retorno en el contexto del paradigma de pre-escucha de Posner (Cueing paradigm), que indica que esta inhibición opera junto a los retrasos que se manifiestan en la atención posterior hecha sobre zonas atendidas con anterioridad, donde se implica importante actividad neural en la corteza parietal (corriente dorsal).

Glimcher (2009) hace referencia a otro modelo implicado en las tareas de reconocimiento visual: se trata del modelo de decisión frontoparietal para los movimientos oculares sacádicos, el cual derivó de diversos resultados que fundamentan la idea de que la presencia de recompensas explícitas impacta en la manera en que se mueven los ojos en el ejercicio de búsqueda de un determinado estímulo. En términos de áreas cerebrales implicadas, destaca la zona LIP, dado que en esta área se hace un procesamiento de la señal de recompensa o refuerzo positivo, donde adicionalmente se genera una modulación que implica un aprendizaje que redunda en una búsqueda por la vía ocular más eficaz (esto está amparado en conclusiones de estudios hechos con primates).

Otros estudios, realizados con otras especies animales, también han aportado en la construcción de modelos que explican mecanismos atencionales relacionados con procesamientos perceptuales de orden visual. Es el caso de modelos fundamentados en el estudio de la retina de las ranas, entre los que destaca el bug detector model, que fue propuesto por Barlow (1972) y verificado por Lettvin,
Maturana, McCulloch, y Pitts (1959), y que dio emergencia a una teoría desarrollada por Konorski, que de manera esencial sugiere que son definitivas las redes basadas en representaciones explícitas de conceptos más allá de la modalidad sensorial. "... existirían aristocracias neuronales de cuya cooperación o competencia (sesgada por la actividad ejecutiva, motora, sensorial o emocional) podría resultar el comportamiento perceptivo, y, por lo tanto, cognitivo del individuo" (Caputi \& Rudelli, 2014, p. 252). Este modelo implica la existencia de redes neuronales, en las cuáles hay células, que como propiedad emergente se activan o disparan específicamente ante un estímulo (de cualquier modalidad), siempre y cuando "dicho estímulo tenga como característica un conjunto mínimo de rasgos específicos de un objeto" (Caputi \& Rudelli, 2014, p. 252).

En los seres humanos, neuronas en el núcleo subtalámico muestran una actividad selectiva durante los movimientos sacádicos que operan en función de un direccionamiento de la mirada hacia objetivos específicos. También, en seres humanos se ha detectado que varias áreas del cerebro están relacionadas con los movimientos oculares, como por ejemplo el surco intraparietal. Así mismo, se ha encontrado que varias neuronas que juegan un papel en la selección de objetivos visuales se encuentran en el colículo superior. Este hecho es significativo, especialmente si se toma en consideración que estas células justamente presentan una importante actividad cuando se están buscando objetivos visuales específicos en comparación a cuando se presentan estímulos distractores dentro de un diseño experimental. De conformidad con lo que reseñan Himmelbach, Erb, y Kartath (2007) y White, Gegenfurtner, y Kerzel (2005), así hubiese pocas aportaciones en el estudio del colículo superior en asocio a la generación de movimientos sacádicos en los seres humanos, resulta plausible correlacionar la actividad de esta parte del cerebro con movimientos sacádicos reflexivos, los cuales son activados de manera exógena, bien sea por la aparición de un estímulo periférico, o bien por la desaparición de un estímulo de fijación. En la Figura 2, se presenta un 
esquema en el que se relacionan los modelos acá reseñados, donde se pueden advertir las nociones fundamentales involucradas:

Figura 2

Esquema ilustrativo de modelos implicados en el rastreo visual de imágenes

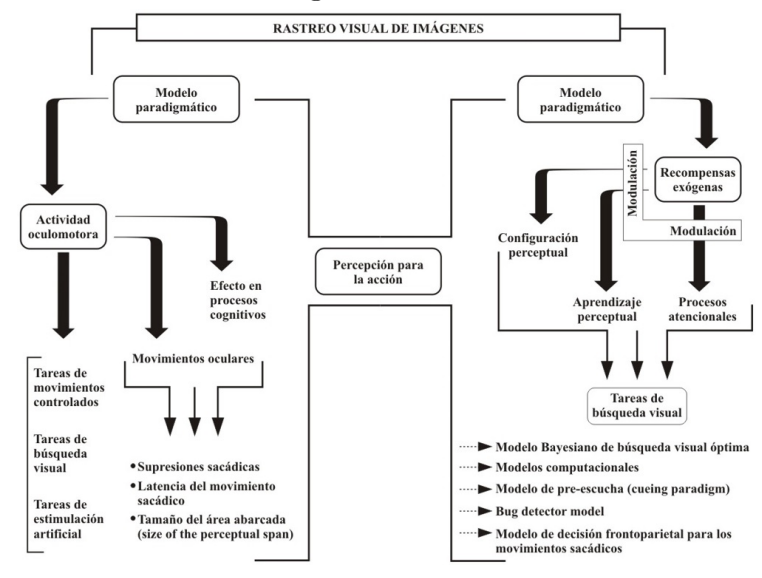

\section{Conclusiones}

En conformidad con lo manifestado, puede convenirse que los estímulos visuales se convierten en representaciones mentales gracias a un complejo intrincamiento de actividades intrapsíquicas, que permiten que el ser humano distinga una imagen desde la aprehensión mental de categorías tales como la forma, el tamaño, el movimiento, la profundidad, el color y la textura, entre otras. En el campo visual, habrán de presentarse estas categorías, que son relativas a los estímulos visuales que grupos independientes de neuronas logran integrar, suscitando lo que bien puede denominarse mecanismos de integración (Kandel, Schwartz, \& Jessell, 2008). No dejar de lado que las maneras de coordinar los movimientos oculares cambian cuando recompensas explícitas están relacionadas con la posición final del ojo. También agregar que los procesos de aprendizaje perceptual hacen parte del conjunto de factores inmersos en la ejecución de tareas de búsqueda visual, y que los movimientos sacádicos se configuran también de conformidad con los puntos de fijación iniciales (Trommershäuser et al., 2009); desde la lectura de los tiempos de respuesta de los movimientos sacádicos se evidencia que el procesamiento de la información sensorial y el inicio del movimiento ocular se producen, en buena medida, en paralelo. Así, la latencia sacádica tiene una fuerte influencia de la presencia (o ausencia) de un punto de fijación visual, justo como se ha encontrado en múltiples estudios hechos sobre modulación semántica de la percepción visual bajo el paradigma de las figuras ambiguas o biestables (Gale \& Findlay, 1983; Hsiao et al., 2012).

Para cerrar, poner de manifiesto que las tareas de búsqueda visual, ligadas a los procesos implicados en los movimientos oculares, se constituyen en una herramienta supremamente útil para evaluar los procesos de recopilación, integración y procesamiento de la información, valiéndose de pruebas experimentales más bien simples de laboratorio, lo que, a la postre, permite producir conocimiento en condiciones similares a las que en efecto está expuesto el ser humano en su cotidianidad. En esa línea de ideas, se concatena que las tareas de búsqueda visual seguirán siendo actividades esenciales en el ámbito de la investigación para entender el comportamiento humano en diversos dominios industriales como la aeronavegación, la conducción de automotores, la fijación de objetivos militares, etc., como lo remarcan Yu y Chan (2013).

Sin lugar a dudas, el desarrollo de la investigación referida a procesos neuropsicológicos y cognitivos seguirá encontrando en el paradigma de las tareas de búsqueda visual un punto de apalancamiento esencial, al punto que futuros investigadores podrán abonar en la construcción de nuevo conocimiento, esto en consonancia con la innovación que en el plano tecnológico emerja para facilitar la instrumentalización y la manera de obtener la información en estudios que tengan por objeto la complejidad que supone la percepción visual humana. 


\section{Referencias}

Barlow, H. B. (1972). Single units and sensation: a neuron doctrine for percetual psychology? Perception, 1 (4), 371-394. https://doi.org/10 $.1068 / \mathrm{p} 010371$

Brouwer, A. M., Reuderink, B., Vincent, J., van Gerven, M. A., \& van Erp, J. B. (2013). Distinguishing between target and nontarget fixations in a visual search task using fixation-related potentials. Journal of Vision, 13(3), 17. https://doi.org/10.1167/1 3.3.17

Caputi, A., \& Rudelli, R. (2014). Procesamiento sensorial y percepción. En D. Redolar (Ed.), Neurociencia cognitiva (pp. 231-256). Madrid: Editorial Médica Panamericana.

Carlson, N. R. (2006). Fisiología de la conducta. Madrid: Pearson Educación.

Coren, S., Ward, L. M., \& Enns, J. T. (2001). Sensación y percepción. Ciudad de México: McGraw-Hill.

Dandekar, S., Ding, J., Privitera, C., Carney, T., \& Klein, S. A., (2012). The fixation and saccade p3. PLoS One, 7(11), e48761. http s://doi.org/10.1371/journal.pone.0048761

Found, A., \& Müller, H. J. (1996). Searching for unknown feature targets on more than one dimension: Investigating a "dimensionweighting" account. Attention, Perception, E) Psychophysics, 58(1), 88-101. https:// doi.org/10.3758/BF03205479

Gale, A. G., \& Findlay, J. M. (1983). Eyemovement patterns in viewing ambiguous figures. En R. Groner (Ed.), Eye movements and psychological functions: international views (pp. 145-168). Hillsdale, NJ: LEA.

Geyer, T., Müller, H. J., \& Krummenacher, J. (2006). Cross-trial priming in visual search for singleton conjunction targets: Role of repeated target and distractor features. Perception Eु Psychophysics, 68(5), 736-749. https://doi.org/10.3758/BF03193697

Glimcher, P. W. (2009). Choice: towards a standars back-pocket model. En P. Glimcher, \& E. Fehr (Eds.), Neuroeconomics: Decision Making and The
Brain (pp. 501- 519). Londres: Academic Press.

Himmelbach, M., Erb, M., \& Kartath, H. (2007). Activation of superior colliculi in humans during visual exploration. BMC Neuroscience, 8, 66. https://doi.org/10.118 6/1471-2202-8-66

Hochberg, J., \& Peterson, M. (1987). Piecemeal organization and cognitive components in object perception: Perceptually coupled responses to moving objects. Journal of Experimental Psychology: General, 116(4), 370-380.

Hsiao, J., Chen, Y., Spence, Ch., \& Yeh, S. (2012). Assessing the effects of audiovisual semantic congruency on the perception of a biestable figure. Consciousness and Cognition, 21(2), 775-787. https://doi.org/1 0.1016/j.concog.2012.02.001

Kamienkowski, J. E., Ison, M. J., Quiroga, R. Q., \& Sigman, M. (2012). Fixation-related potentials in visual search: a combined EEG and eye tracking study. Journal of Vision, 12(7), 4. https://doi.org/10.1167/12.7.4

Kandel, E., Schwartz, J., \& Jessell, T. (2008). Neurociencia y conducta. Madrid: Prentice Hall.

Kazai, K., \& Yagi, A. (1999). Integrated effect of stimulation at fixation points on EFRP (eye-fixation related brain potentials). International Journal of Psychophysiology, 32(3), 193-203. https://doi.org/10.1016/SO 167-8760(99)00010-0

Kornmeier, J., \& Bach, M. (2012). Ambiguous figures - What happens in the brain when perception changes but not the stimulus. Frontiers in Human Neuroscience, 6, 1-23. h ttps://doi.org/10.3389/fnhum.2012.00051

Leopold, D., \& Logothetis, N. (1999). Multistable phenomena: changing views in perception. Trends in cognitive sciences, 3(7), 254-264. https://doi.org/10.1016/S1364-66 13(99)01332-7

Lettvin, J., Maturana, H., McCulloch, W., \& Pitts, W. (1959). What's the frog's eye tells the frog's brain? Proceedings of the Institute of Radio Engineers, IRE, 47(11), 
1940-1951. https://doi.org/10.1109/JRPRO C.1959.287207

Long, G. M., \& Moran, C. (2007). How to keep a reversible figure from reversing: Teasing out top-down and bottom-up processes. Perception, 36(3), 431-445. https://doi.org/1 $0.1068 / \mathrm{p} 5630$

Long, G. M., \& Olszweski, A. D. (1999). To reverse or not to reverse: When is an ambiguous figure not ambiguous? American Journal of Psychology, 112(1), 41-71. http:// dx.doi.org/10.2307/1423624

Long, G. M., \& Toppino, T. C. (2004). Enduring interest in perceptual ambiguity: alternating views of reversible figures. Psychological Bulletin, 130(5), 748-768. http ://dx.doi.org/10.1037/0033-2909.130.5.748

Long, G. M., Stewart, J. A., \& Glancey, D. E. (2002). Configural biases and reversible figures: Evidence of multilevel grouping effects. American Journal of Psychology, 115(4), 581-607. http://dx.doi.org/10.2307 $/ 1423528$

Long, G. M., Toppino, T. C., \& Kostenbauder, J. F. (1983). As the cube turns: Evidence for two processes in the perception of a dynamic reversible figure. Perception $\mathbb{E}$ Psychophysics, 34(1), 29-38. https://doi.org/10.3758/BF03 205893

Long, G. M., Toppino, T. C., \& Mondin, G. W. (1992). Prime time: Fatigue and set effects in the perception of reversible figures. Perception $\mathcal{E}$ Psychophysics, 52 (6), 609-616. https://doi.org/10.3758/BF03211697

Maarek, P. (2009). Marketing político y comunicación. Claves para una buena información política. Barcelona: Paidós.

Maljkovic, V., \& Nakayama, K. (2000). Priming of pop out: III. A short-term implicit memory system beneficial for rapid target selection. Visual Cognition, 7(5), 571-595. h ttps://doi.org/10.1080/135062800407202

Marr, D. (1982). Vision. New York: W.H. Freeman and Company.

Müller, H. J., Heller, D., \& Ziegler, J. (1995). Visual search for singleton feature targets within and across feature dimensions.
Perception Eु Psychophysics, 57(1), 1-17. htt ps://doi.org/10.3758/BF03211845

Najemnik, J., \& Geisler, W. (2008). Eye movement statistics in humans are consistent with an optimal search strategy. Journal of Vision, 8(3), 1-14. https://doi.org/ 10.1167/8.3.4

Pires, A., Vásquez, A., Carboni, A., \& Maiche, A. (2014). Percepción visual. En D. Redolar (Ed.), Neurociencia cognitiva (pp. 257-286). Madrid: Editorial Médica Panamericana.

R. H. Wurtz, \& M. E. Goldberg (Eds.). (1989). The Neurobiology of Saccadic Eye Movements (Reviews of Oculomotor Research Vol. 3). Amsterdam: Elsevier.

Rayner, K. (1998). Eye movements in reading and information processing: 20 years of research. Psychological Bulletin, 124(3), 372-422. http://doi.org/10.1037/0033-2909 .124 .3 .372

Roca, E. M., Rosselló, J., Maiche, A., García, D. T., \& Roberts, M. N. (2008). Modelos teóricos y neurociencia cognitiva de la percepción. En J. Tirapú, F. Maestú, \& M. Ríos (Eds.), Manual de neuropsicologia (pp. 59-96). Barcelona: Viguera Editores.

Shah, D. S., Prados, J., Gamble, J., De Lillo, \& Gibson, C. (2013). Sex differences in spatial memory using serial and search tasks. Behavioural Brain Research, 257, 90-99. htt ps://doi.org/10.1016/j.bbr.2013.09.027

Thomas, N., \& Pare, M. (2007). Temporal processing of saccade targets in parietal cortex area LIP during visual search. Journal of Neurophysiology, 97(1), 942-947. https:// doi.org/10.1152/jn.00413.2006

Treisman, A. (1986). Features and objects in visual processing. Scientific American, 255(5), 114-125. https://doi.org/10.1038/sc ientificamerican1186-114B

Treisman, A. (1988). Features and objects: The fourteenth Bartlett memorial lecture. The Quarterly Journal of Experimental Psychology, 40(2), 201-237. https://doi.org/10.1080/02 724988843000104

Treisman, A. (1991). Search, similarity, and integration of features between and within dimensions. Journal of 
Experimental Psychology: Human Perception and Performance, 17(3), 652-676.

Trommershäuser, J., Glimcher, P., \& Gegenfurtner, K. (2009). Visual processing, learning and feedback in the primate eye movement system. Trends in Neurosciences, 32 (11), 583-590. https://doi.org/10.1016/j.t ins.2009.07.004

von Grünau, M. W., Wiggin, S., \& Reed, M. (1984). The local character of perspective organization. Perception $\mathbb{E}$ Psychophysics, 35(4), 319-324. https://doi.org/10.3758/BF 03206335

Weidner, R., Pollmann, S., Müller, H. J., \& von Cramon, D. Y. (2002). Top-down controlled visual dimension weighting: An event-related fMRI study. Cerebral Cortex, 12 (3), 318-328. https://doi.org/10.1093/cer cor $/ 12.3 .318$

White, B. J., Gegenfurtner, K. R. \& Kerzel, D. (2005). Effects of structured nontarget stimuli on saccadic latency. Journal of Neurophysiology, 93(6), 3214-3223. https:// doi.org/10.1152/jn.01104.2004

White, B. J., Stritzke, M., \& Gegenfurtner, K. R. (2008). Saccadic facilitation in natural backgrounds. Current Biology, 18(2), 124-128. https://doi.org/10.1016/j.c ub.2007.12.027

$\mathrm{Xu}$, J., \& Yue, S. (2014). Mimicking visual searching with integrated top down cues and low-level features. Neurocomputing, 133, 1-17. https://doi.org/10.1016/j.neuco m.2013.11.037

Yu, R., \& Chan, A.H.S. (2013). Visual search time in detection tasks with multiple targets: Considering change of the effective stimulus field area. International Journal of Industrial Ergonomics, 43(4), 328-334. https ://doi.org/10.1016/j.ergon.2013.04.010

\section{Notas}

* Artículo de investigación. Estudio financiado por la Universidad de Bogotá Jorge Tadeo Lozano. 